\title{
AN IMPROVED PAPR REDUCTION TECHNIQUE FOR UNIVERSAL FILTER MULTI-CARRIER MODULATION
}

\author{
Pooja RANI, Silki BAGHLA, Himanshu MONGA \\ Department of Electronics and Communication Engineering, J.C.D.M. College of Engineering, Sirsa, India, \\ E-mail: poojamehta0193@gmail.com, silky.er@gmail.com,himanshumonga@gmail.com
}

\begin{abstract}
Universal Filter Multi-Carrier (UFMC) modulation is a potential candidate for next generation wireless communication due to its advantages like better sub carrier separation and less complexity. Higher peak to average power ratio (PAPR) affects the performance of UFMC and suitable PAPR reduction technique is required to mitigate the same. In this work, an improved PAPR reduction technique (CFC-UFMC) has been proposed for UFMC system. Performance of proposed technique is compared with existing Selective Mapping (SLM), Clipping, Companding and Clipping \& Filtering methods as applied on UFMC. Simulation results show that proposed technique provides better PAPR reduction with variation in design parameters namely FFT Size, Filter Length and Bits per sub carrier as compared with other techniques. Various PAPR reduction techniques have also been compared on the basis of bit error rate.
\end{abstract}

Keywords: UFMC, CFC-UFMC, SLM, FFT and PAPR

\section{INTRODUCTION}

Multicarrier modulation is a method of transmitting data by splitting it into several components so that a higher data transmission rate can be achieved [1]. Many multi carrier modulation techniques have been developed by researchers to support higher data transmission rates and UFMC, FBMC and OFDM are some of them [2]. UFMC is one of the multicarrier modulation techniques which are being considered as potential candidates for next generation wireless communication [3]. In UFMC input data stream is divided into number of sub carriers and groups of these sub carriers are formed to make sub bands which are filtered through pulse shaping filters before transmission.

Like other multicarrier modulation techniques it also suffers from the drawback of higher peak to average power ratio [4]. There are a number of PAPR reduction techniques like clipping, selective mapping, companding and clipping with filtering are available which were originally introduced for OFDM systems and cannot be directly implemented on UFMC due to different frame structures. In this work, we have made an effort to make some of the available PAPR reduction techniques suitable for UFMC system and a comparative analysis is done to find the most effective technique and a new improved PAPR reduction technique has been implemented.

The remainder of this paper is organized as follows: In section 2, the considered UFMC system model is presented. Section 3 is devoted to the high Peak to average power ratio and PAPR reduction techniques. Section 4 reports simulation setup, including the comparative analysis of different PAPR reduction techniques. Finally, conclusions are drawn in Section 5.

\section{UFMC SYSTEM MODEL}

Multicarrier modulations are based on the principle of splitting data into several components and send these over many different sub channels, so that a higher data transmission rate can be achieved. UFMC system is also based on the principle of frequency division multiplexing
(FDM), in which input data stream is divide into several lower rate sub-streams. Basically, it provides better sub carrier separation like FBMC and less complex system like OFDM. Filter bank multicarrier (FBMC) filters the signal on per subcarrier basis i.e. each sub carrier is assigned its own filter [5] which provides better sub carrier separation but on the other hand use separate filer for each sub carrier, makes the system more complex while orthogonal frequency division multiplexing (OFDM) filters the signal on single shot i.e. single filter is used for complete signal this provides a simple system but sub carrier separation is also poor [6]. UFMC (Universal filter multicarrier) can be seen as a compromise between OFDM and FBMC, it groups subcarriers to form subbands, which are then filtered individually as compared with single subcarrier or the complete band [7].

Fig. 1 shows block diagram of UFMC, input data stream is divided into sub-streams, and then these sub carriers are grouped to form sub-bands. These sub bands and then a pulse shaping filter with smooth edges is used on each sub band that leads to substantial reduction in outof-band transmission [8]. In this way, it minimizes the harmful interference from adjacent sub channels of the neighboring resource block.

The generated output can be given as:

$\mathrm{x}_{\mathrm{k}}=\sum_{\mathrm{p}=1}^{\mathrm{B}} \mathrm{F}_{\mathrm{p}, \mathrm{k}} \mathrm{V}_{\mathrm{p}, \mathrm{k}} \mathrm{X}_{\mathrm{p}, \mathrm{k}}$

The overall bandwidth and the total number of subcarriers are divided into number of sub bands. Now, the input data stream of $\mathrm{k}^{\text {th }}$ user, $x_{k}$ is divided into multiple sub streams denoted by $X_{p . k}$ for $\mathrm{p} \epsilon\{1,2,3 \ldots \ldots \ldots . B\}$. Here ' $\mathrm{B}$ ' is total number of sub streams or sub bands. A sub band in UFMC may also correspond to Physical Resource Block (PRB) in LTE.

Then, the signal of each sub band is applied to individual $\mathrm{N}$ point IFFT represented by matrix ' $\mathrm{V}$ '. The output of IFFT is converted to serial form and applied to respective filter represented by matrix ' $F$ '. ' $F$ ' is a Toeplitz matrix, composed of the filter impulse response, performing the linear convolution in equation (1). For $\mathrm{p}^{\text {th }}$ 
sub band, (where $\mathrm{p}$ varies from 1 to B) $X_{p, k}, V_{p, k}$ and $F_{p, k}$ represents data blocks, IFFT matrix and filter respectively. For filter length ' $\mathrm{L}$ ' and IFFT matrix of size ' $N$ ', the symbol $\mathrm{x}_{\mathrm{k}}$ of duration $\mathrm{N}+\mathrm{L}-1$ is generated at the output of transmitter section.

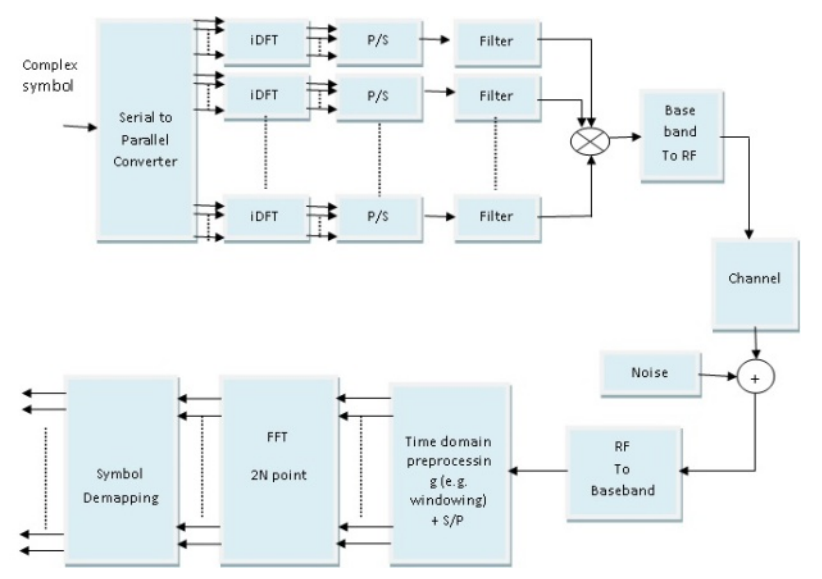

Fig. 1 UFMC System Model

UFMC system is more robust to transceiver imperfections such as carrier frequency offset (CFO) and timing offset (TO) and it is a low-cost low-complexity system. In addition to the benefits in performance and the lower out of band transmission, the most important advantage of the UFMC system over OFDM system is the design flexibility, which enables the system to adapt to the requirements of specific user, service types and channels by adjusting the sub band filter and system parameters [9]. The filter length is a key parameter affecting the system performance in different ways. A longer filter not only provides lower out of band emission, but also results in better frequency localization and makes the system more robust to synchronization errors and multipath fading channels [10]. However, a longer filter also causes several drawbacks including more frequency selective filter response reducing transmission efficiency in the time domain. So, it is clear that by selecting proper filter length and system parameters an efficient and better system for next generation wireless communication can be designed.

\section{PEAK TO AVERAGE POWER RATIO AND PAPR REDUCTION TECHNIQUES}

The PAPR is the relation between the maximum power of a sample in a given by transmit symbol divided by the average power of that symbol. PAPR occurs when in a multicarrier system the different sub-carriers are out of phase with each other [11]. At each instant they are different with respect to each other at different phase values. When all the points achieve the maximum value simultaneously, this will cause the output envelope to suddenly increase which causes a 'peak' in the output. There are a large number of independently modulated subcarriers in multicarrier system, and when they are added up coherently for transmission purpose give a large peak value which is very large as compared to average value of the sample. The ratio of the peak to average power value is termed as Peak-to-Average Power Ratio and it can be given as:
$\operatorname{PAPR}=\left(\frac{\max \left\{|x[n]|^{2}\right\}}{E\left\{|x[n]|^{2}\right\}}\right)$

Where, $|\mathrm{x}[n]|$ is the amplitude of $\mathrm{x}[\mathrm{n}]$ and $\mathrm{E}$ denote the expectation of the signal.

This higher PAPR causes saturation in power amplifier which produces inter modulation products among sub bands and also increases out of band radiation [12]. So, to make system performance efficient it is necessary to reduce peak to average power ratio. There are many techniques for PAPR reduction, which were originally designed for OFDM systems, some of them can be described as:

\subsection{Selected Mapping (SLM)}

The basic idea behind this scheme is to generate $\mathrm{N}$ statistically independent data frames representing the same information and frame with lowest PAPR is selected for transmission, because of the varying assignment of data to the transmit signal this principle is called as selected mapping [13].

\subsection{Clipping and filtering}

This is one of the simplest techniques for PAPR reduction. The principle is to define a clipping level for data transmission above which the input signal is clipped off and peaks of signal are reduced [14]. Let, there is a signal $x[n]$ which is to be transmitted and $x_{c}[n]$ is its clipped version which can be denoted as:

$x_{c}[n]= \begin{cases}-A & x[n] \leq-A \\ x[n] & |x[n]|<A \\ A & x[n] \geq A\end{cases}$

Where, A is the clipping level. Clipping operation reduces PAPR at cost of higher BER and it also produces in-band and out of band radiation in signal. This out of band radiation can be reduced by using filtering after clipping but BER performance gets degraded.

\subsection{Tone Reservation}

In this scheme, some subcarriers are reserved within the transmitted bandwidth and appropriate value is assigned to these reserved tones. These reserved subcarriers don't carry any data information, are only used for reducing PAPR. This method is called Tone Reservation [15]. Main advantage of TR is that it does not introduce any distortion to the data bearing tones and no side information is required. But it increases average energy per bit which might reduce the BER performance and spectral efficiency is also reduced due to tone reservation.

\subsection{Companding}

Companding is an easy and less complex method of PAPR reduction, the basic idea was to expand the small signal in transmitter section and compression is carried out at receiver side. In this method value of small signals is enlarged while peaks remain unchanged due to which 
average power is increased and peak to average ratio is reduced.

Although all these techniques were originally design for Orthogonal Frequency Division Multiplexing and due to different frame structure of FBMC and UFMC some of these cannot be directly applied on these modulation techniques. Therefore it is necessary to consider the different frame structure of these modulation schemes while applying PAPR reduction techniques [16].

\subsection{Combining Clipping and Filtering with Companding (Proposed CFC-UFMC Technique)}

In this work, an improved PAPR reduction technique CFC-UFMC has been proposed. It is the combination of Clipping and Filtering with Companding. Fig. 2 shows step by step procedure for implementation of proposed technique.

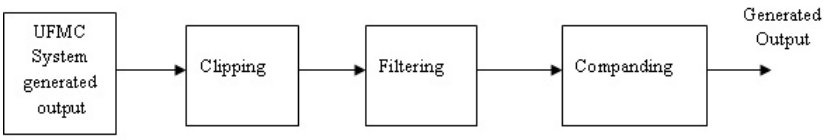

Fig. 2 CFC-UFMC PAPR Reduction Technique

Step 1: Complex symbols are generated by UFMC modulator system.

Step 2: Define a clipping level above which the input signal is clipped of to remove picks.

Step 3: Out of band radiations of clipped signal are removed by Filtering.

Step 4: At transmitter, small signals are enlarged while peaks remain unchanged due to which average power is increased.

Step 5: At receiver reverse operation is performed and compression of transmitted signal is carried out.

To improve the PAPR reduction of UFMC signal we have proposed CFC-UFMC technique. The basic idea is to clip the output signal generated by a UFMC modulator to a predefined level ' $A$ ' above and below the average value. Here, clipping level is defined $4 \mathrm{~dB}$ above and below the average. This clipped signal is then passed through a filter section whose filter length can be varied and for our consideration we have taken three values $=43,63$ and 83 . Companding is done to expand the small signal in transmitter section and compression is carried out at receiver side, we are using $\mu$ law companding with $\mathrm{M}=$ 255. Combination of these three techniques may provide a large amount of reduction in peak to average power ratio of the system which is desired for making a modulation system more effective.

\section{SIMULATION SETUP}

The proposed PAPR reduction technique has been implemented on UFMC modulation technique. Table 1 provides the simulation set up used in this work. Here, we have used Dolph-chebyshev filter for simulation of UFMC system with QAM as baseline modulation scheme.
Table 1 Simulation Parameters for UFMC system

\begin{tabular}{|l|l|}
\hline \multicolumn{1}{|c|}{ Parameter } & Value \\
\hline Filter & Dolph-chebyshev filter \\
\hline Filter Length & $43,63,83$ \\
\hline Modulation- Scheme & QAM \\
\hline FFT Size & $256,512,1024$ \\
\hline Bits Per Sub Carrier & $2,4,6$ \\
\hline Sub Band Size & 20 \\
\hline Number of Sub Bands & 10 \\
\hline
\end{tabular}

MATLAB platform is used to perform the comparison.

\section{RESULTS AND DISCUSSION}

The proposed CFC-UFMC technique has been evaluated with variation in filter length, FFT size and Bits per sub carrier. The performance of proposed technique is also compared with 4 other techniques namely Selective Mapping (S-UFMC), Clipping technique (C-UFMC), Companding (Comp UFMC), Clipping and Filtering (CFUFMC). The work is further extended and effect of these PAPR reduction techniques has been studied on the bit error rate of the system.

\subsection{Effect of FFT size}

FFT size of any modulation system has a great impact on its performance. In this work, we have examined effect of FFT size on performance of different PAPR reduction techniques as applied with UFMC system. Fig. 3 provides the impact of variation in FFT Size on Different PAPR reduction techniques.

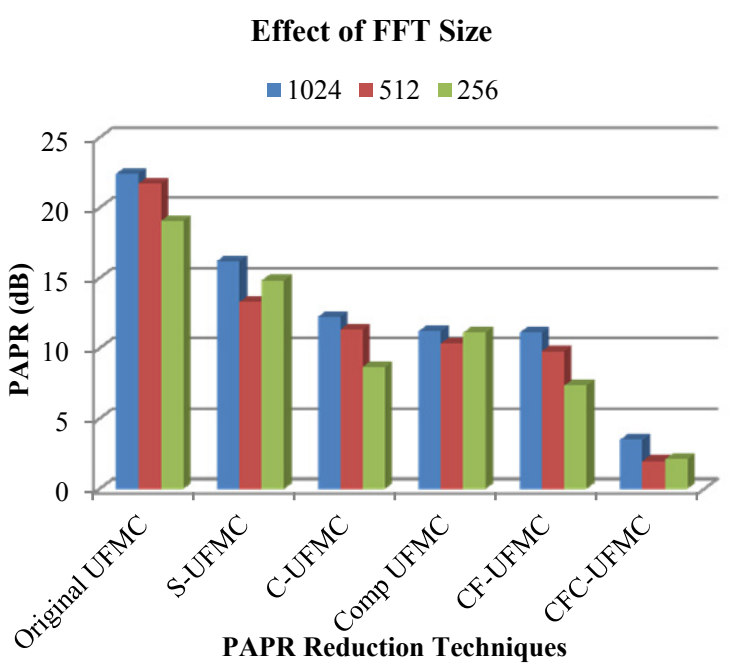

Fig. 3 Effect of FFT size on different PAPR reduction Techniques

It is clear from Fig. 3 that, FFT Size has a significant impact on the PAPR distribution of UFMC signals. Hence, the PAPR increases for large values of FFT Size. This is due to the reason that overlapping of symbols increases with increase in FFT size which results in increase in PAPR value. Likewise with short FFT lengths, the PAPR decreases. This holds true for all the PAPR reduction techniques considered in this work. 
The amount of PAPR reduction provided by CFCUFMC varies from $84 \%$ to $90 \%$ with variation in FFT size and its maximum at moderate value (512-point) of FFT size.

\subsection{Effect of Bits per Sub carrier}

Bits per sub carrier (BPS) and modulation order are inter-related to each other. Values of 2, 4, and 6 Bits per sub carrier results in modulation order of 4,16 , and 64 respectively. Table 2 shows effect of bits per sub carrier on different PAPR reduction techniques.

Table 2 Effect of Bits per Sub-Carrier

\begin{tabular}{|c|c|c|c|c|c|c|}
\hline Bits & \multicolumn{6}{|c|}{ PAPR (dB) } \\
\hline $\begin{array}{l}\text { Per } \\
\text { Sub } \\
\text { carrie } \\
\text { r }\end{array}$ & $\begin{array}{l}\text { Origin } \\
\text { al } \\
\text { UFMC }\end{array}$ & $\begin{array}{l}\text { S- } \\
\text { UFM } \\
\text { C }\end{array}$ & $\begin{array}{l}\text { C- } \\
\text { UFM } \\
\text { C }\end{array}$ & $\begin{array}{l}\text { Com } \\
\text { p } \\
\text { UFM } \\
\text { C }\end{array}$ & $\begin{array}{l}\text { CF- } \\
\text { UFM } \\
\text { C }\end{array}$ & $\begin{array}{l}\text { CF } \\
\text { C- } \\
\text { UF } \\
\text { MC }\end{array}$ \\
\hline 2 & 22.44 & 16.2 & 12.2 & 11.2 & 11.1 & 3.50 \\
\hline 4 & 19.58 & 21.1 & 17.7 & 11.1 & 6.79 & 1.33 \\
\hline 6 & 21.62 & 21.0 & 25.8 & 10.5 & 9.41 & 1.65 \\
\hline
\end{tabular}

It can be concluded that PAPR of UFMC is minimum with CFC-UFMC technique and it provides better results than other PAPR reduction techniques considered in this work. The effectiveness of CFC-UFMC increased from $84 \%$ to $93 \%$ with variation in Bits per sub carrier and it is $93 \%$ with 4 bits per sub carrier.

\subsection{Effect of Filter Length}

The filter length is a key parameter affecting the PAPR value of a system. A longer filter not only provides lower out of band emission, but also results in better frequency localization However, a longer filter also causes several drawbacks including more frequency selective filter response reducing transmission efficiency in the time domain. The results obtained by variation in Filter Length are given in Fig. 4.

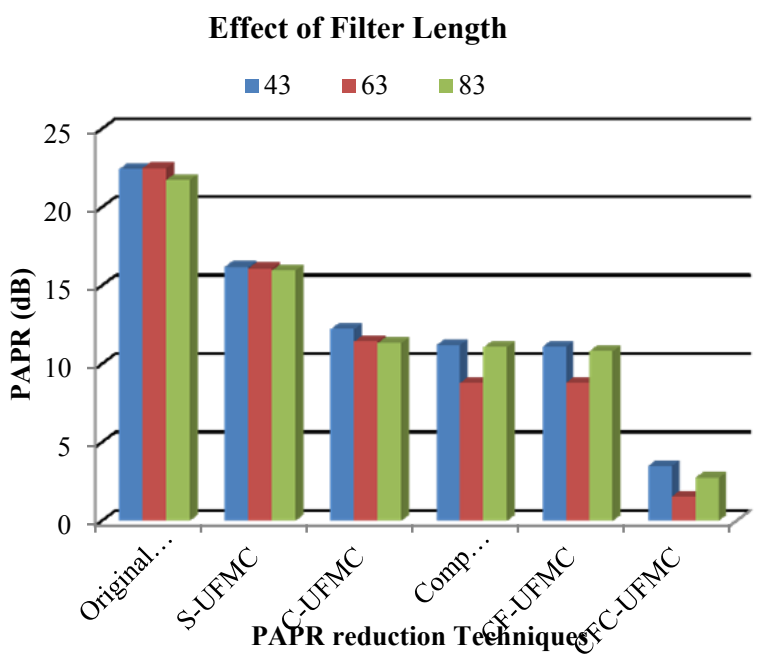

Fig. 4 Effect of Filter length on Various PAPR reduction Techniques
We have considered filter length 43, 63 and 83 to evaluate the performance of CFC-UFMC. The results obtained by the simulation have shown that the performance of proposed method is better than other PAPR reduction techniques.

Fig. 4 clarifies that, with variation in filter length, the performance of proposed technique varies but it is better than other PAPR reduction techniques considered in this work.

CFC-UFMC provides a large amount of PAPR reduction and effectiveness of the scheme increased from $84 \%$ to $93 \%$ with variation in filter length. It provides maximum amount of PAPR reduction with filter length 63.

\subsection{Bit Error Rate}

Bit error rate can be defined as the ratio of bits that have errors relative to the total number of bits received in a transmission. In digital transmission received bits of a data stream over a communication channel get altered due to noise, interference or distortion. Bit error rate is a performance measure of any communication system and defined in percentage. The definition of bit error rate can be given as:

$$
\mathrm{BER}=\frac{\text { Number of errors }}{\text { Total number of transmitted bits }}
$$

In this paper, effect of PAPR reduction techniques on BER performance of system has been studied. It has been evaluated that with the reduction of PAPR, bit error rate the system starts increasing. Fig. 5 shows the bit error rate of UFMC system, without and with PAPR reduction techniques.

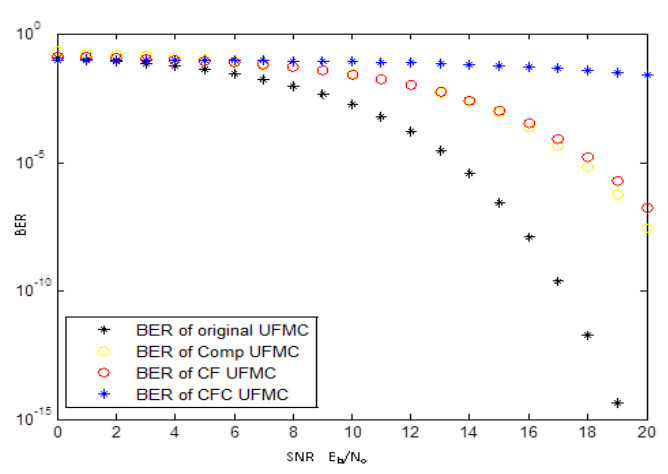

Fig. 5 Comparison of BER with different PAPR reduction Techniques

Here, a comparison of conventional UFMC signal has been done with Comp-UFMC, CF-UFMC and CFCUFMC on the basis of BER. For this comparison SNR range is taken from 0 to 20 and BER value of original signal is nearly equal to $10^{-3}$ but when we apply PAPR reduction techniques it starts decreasing, if we compare these values with PAPR reduction of the techniques it is clear that higher the PAPR reduction lower the BER performance. So, there is a tradeoff between PAPR and $\mathrm{BER}$ of the PAPR reduction techniques. 


\section{CONCLUSION AND FUTURE SCOPE}

In this work, a new PAPR reduction technique CFCUFMC has been simulated and compared with the existing S-UFMC, C-UFMC, Comp-UFMC and CF-UFMC techniques. It has been concluded that proposed technique of PAPR reduction technique provides better results as compared with other existing techniques. Effect of various design parameters on different PAPR reduction techniques has also been evaluated and it is observed that with all design parameters, CFC-UFMC provides better PAPR reduction among all the PAPR reduction techniques considered in this work. Effect of these PAPR reduction techniques has been evaluated on the performance of BER of system and it has been evaluated that there is a tradeoff between BER and PAPR. By using CFC-UFMC technique PAPR reduces to a great extent but BER performance get worst. In future, we will focus on implementing more reliable and efficient PAPR reduction technique for UFMC.

Received October 24, 2017, accepted February 14, 2018

\section{REFERENCES}

[1] BINGHAM, J. A. C.: Multi carrier modulation for data transmission: An idea whose time has come, IEEE Commun. Mag., 28 (5), pp. 5-14, 1990.

[2] ROHDE \& SCHWARZ: Application Note 5G waveform candidates, 2016.

[3] WUNDER, G. - JUNG, P. - KASPARICK, M. WILD, T. - SCHAICH, F. - CHEN, Y. - BRINK, S. - GASPAR, I. - MICHAILOW, N. - FESTAG, A. MENDES, L. - CASSIAU, N. - KTENAS, D. DRYJANSKI, M. - PIETRZYK, S. - EGED, B. VAGO, P. - WIEDMANN, F.: 5GNOW: nonorthogonal, asynchronous waveforms for future mobile applications, IEEE Communications Magazine, Vol. 52, No. 2, pp. 97-105, Feb. 2014.

[4] RANI, P. - BAGHLA, S. - MONGA, H.: Performance Evaluation of Multi carrier modulation techniques for next genration wireless systems, Vol. 8, No. 2, May-June 2017.

[5] SCHAICH, F. - WILD, T.: Waveform contenders for 5G; OFDM vs. FBMC vs. UFMC, in International Symposium on Communications, Control and Signal Processing (ISCCSP), pp. 457460, May 2014.

[6] FARHANG-BOROUJENY, B.: OFDM versus Filter Bank Multicarrier, IEEE Signal Process. Mag., Vol. 28, pp. 92-112, May 2011.

[7] VAKILIAN, V. - WILD, T. - SCHAICH, F. - TEN BRINK, S. - FRIGON, J.-F.: Universal-filtered multi-carrier technique for wireless systems beyond LTE, in IEEE Globecom Workshops (GC Wkshps), pp. 223-228, 2013.
[8] CHEN, Y. - SCHAICH, F. - WILD, T.: Multiple access and waveforms for 5G: IDMA and universal filtered multi-carrier, In Proceedings of IEEE $79^{\text {th }}$ Vehicular Technology Conference (VTC Spring), pp. 1-5, 2014.

[9] Changyoung An - Byeongjae Kim - Heung-Gyoon Ryu: Waveform comparison and Nonlinearity sensitivities of FBMC, UFMC and W-OFDM systems, In Proceedings of the $8^{\text {th }}$ International Conference on Networks \& Communications, pp. 83-90, Dec. 2016.

[10] WILD, T. - SCHAICH, F. - CHEN, Y.: 5G Air Interface Design based on Universal Filtered (UF-) OFDM, In Proceedings of the $19^{\text {th }}$ International Conference on Digital Signal Processing, pp. 699704, 20-23 Aug. 2014.

[11] GANGWAR, A. - BHARDWAJ, M.: An Overview: Peak to Average Power Ratio in OFDM system \&its Effect, International Journal of Communication and Computer Technologies, Vol. 1, No. 2, Issue 2, September 2012.

[12] TELLADO, J. - CIOFFI, J. M.: PAPR Reduction in multicarrier transmission system, Ph.D. Thesis, Stanford university, Feb. 9, 1999.

[13] BAUML, R. W. - FISCHER, R. F. H. - HUBER, J. B.: Reducing the peak to average power ratio of multi carrier modulation by selected mapping, IEEE Electronics Letters, Vol. 32, Oct. 1996.

[14] O'NEILL, R. - LOPES, L. B.: Performance of Amplitude Limited Multitone Signals, In Proceedings of IEEE $44^{\text {th }}$ Vehicular Technology Conference, pp. 1675-1679, 1994.

[15] KRONGOLD, B. S. - JONES, D. L.: A new tone reservation method for complex baseband PAR reduction in OFDM system, Acoustics, Speech and signal processing (ICASSP), IEE International conference 13-17 May, 2002, pp. 2321-2324, 2002.

[16] WANG, X. - TJHUNG, T. T. - NG, C. S.: Reduction of peak to average power ratio of OFDM system using a companding technique, IEEE transactions on broadcasting, Vol. 25, Sep. 1999.

[17] ZHANG, L. - IJAZ, A. - XIAO, P. - IMRAN, M. A. TAFAZOLLI, R.: MU-UFMC System Performance Analysis and Optimal Filter Length and Zero Padding Length Design, IEEE transaction on Signal Processing, 30 March, 2016.

[18] DUAN, S. - YU, X. - WANG, R.: Performance Analysis on Filter Parameters and Subbands Distribution of Universal Filtered Multi-Carrier, Springer, 7 April 2017.

[19] ELMAROUD, B. - FAQIHI, A. - ABBAD, M. ABOUTAJDINE, D.: On The Impact Of Prototype Filter Length On The PAPR Reduction Of FBMC Signals, Internatinal Journal of Engineering and Technology (IJET), Vol. 6, No. 4, Aug.-Sep. 2014. 


\section{BIOGRAPHIES}

Pooja Rani received her B. Tech. degree in Electronics and Communication Engineering from Jan Nayak Choudhary Devi Lal College of engineering Sirsa, in 2015. She is currently M. Tech. student of Electronics and Communication Engineering department in Jan Nayak Choudhary Devi Lal College of Engineering, Sirsa. Her primary interest is wireless communication.

Silki Baghla is Assistant Professor in JCDM College of Engineering, Sirsa (Haryana, India). She has done his Masters in Electronics and Communication from IKG Punjab Technical University (Punjab, India) in 2010. Presently she is pursuing Ph.D from IKG Punjab Technical University (Punjab, India). Her area of research included handover methods, wireless communication, image processing and multiple attributes decision making algorithms.
Dr. Himanshu Monga obtained $\mathrm{PhD}$.from Thapar Institute of Engineering and Technology, (TU) Department Of ECE, Patiala, Punjab, INDIA, in the field of Optical networks / coding and Wireless networks. He obtained his bachelor's degree in Electronics \& Communication Engineering from Govt. Engineering College, Amravati University, Maharashtra, India and Master's degree in Electronics and Telecommunication Engineering \& Masters degree in management in Human resource management. Presently, he is working as Director/Principal in Jan Nayak Chaudhary Devi Lal Lal Vidyapeeth, Sirsa. Before this he served as Professor and Dean Research in Lovely Professional University, Phagwara, India. He possesses work experience of 19 plus years in academics and industry/research. He has more than 95 Research papers in international/national conferences. Have more than 22 Thomson Reuter SCI publications to his credit. He is the life member of IEEE, ISTE and reviewer of many renowned journals. He has authored 04 Books/ chapters in renowned books. He successfully completed 06 projects worth Rs 70 Lacks and successfully completed many consultancy projects. 